\title{
A PARYLENE PERIPHERAL NERVE CUFF ELECTRODE WITH INTEGRATED MICROFLUIDICS
}

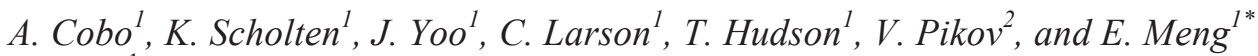 \\ ${ }^{1}$ University of Southern California, Los Angeles, California, USA \\ ${ }^{2}$ GlaxoSmithKline, UK
}

\begin{abstract}
We present a novel micromachined polymer peripheral nerve interface that combines both electrodes and microfluidic channels in an adjustable cuff sized to interface with rat sciatic nerve. Parylene fluidic channels enable targeted delivery of lysing agents and neurotrophic factors to fascicle sites on the nerve surface, in order to induce axonal sprouting towards electrodes. This Lyse-andAttract Cuff Electrode (LACE) uses a simple locking mechanism that is adjustable for close contact with nerves of varying diameters. We demonstrate fully functional cuffs having electrode impedances of $<2 \mathrm{k} \Omega(1 \mathrm{kHz})$ and controlled microfluidic infusion at $<\mu \mathrm{L} / \mathrm{min}$ flow rates.
\end{abstract}

\section{INTRODUCTION}

Neural recording and stimulation technologies are needed to interface with peripheral nervous system (PNS) to create a bidirectional link between the nervous system and artificial devices (prosthesis) for restoration of sensorimotor function (Figure 1) [14]. Electrical interfaces to the peripheral nerves are either extraneural or intrafascicular/intraneural. Minimally invasive extraneural interfaces are placed on the circumference of the nerve which is surrounded by a collagenous sheath (epineurium) that limits access to individual nerve fibers. Improved access to individual fascicles has been achieved by extraneural interfaces that reshape the nerve by flattening it, as is the case of the previously described flat interface nerve electrode (FINE) [5]. However the signal to noise ratio is still restricted by the impedance of the epineurium, and nerve damage can be induced by reshaping.

Intrafascicular/intraneural electrodes gain greater access to individual nerve fibers, improving signal-to-noise ratio, and can selectively target small groups of axons, but are significantly more invasive [6, 7]. Electrodes may either be inserted longitudinally into the nerve and run parallel to nerve fibers, or transversely and run perpendicular to fibers. Of the two methods, transverse electrodes are associated with greater risk of nerve damage. Invasively inserted electrodes are fabricated from materials with elastic moduli several orders of magnitude greater than that of nerve, contributing to chronic tissue response and the degraded recording/stimulation stability over time.

Neither approach has been able to achieve the desired selectivity while minimizing invasiveness, and all prior attempts suffered from a lack of chronic reliability. To overcome these hurdles and achieve a long-term stable interface with high fidelity recordings, we developed a non-invasive approach in which a polymer cuff electrode targets individual fascicles within a nerve by inducing the sprouting of axonal collaterals towards electrodes embedded in the microfluidic channels of the cuff. Axonal sprouting will be selectively induced from the fascicles closest to the microfluidic outlet ports via localized delivery of lysing agents to temporarily disrupt the epineurium, followed by delivery of neurotrophic factors to promote axonal sprouting towards electrodes within the microchannels [8-11]. The design calls for a cuff with adjustable diameter to ensure localized diffusion of chemicals into the epineurium of nerves of varying diameters. This Lyse-andAttract Cuff Electrode (LACE) aims to increase fascicular selectivity and signal-to-noise ratio for recording and/or stimulation without traumatic invasiveness, while providing long-term stability beyond the current state of the art.

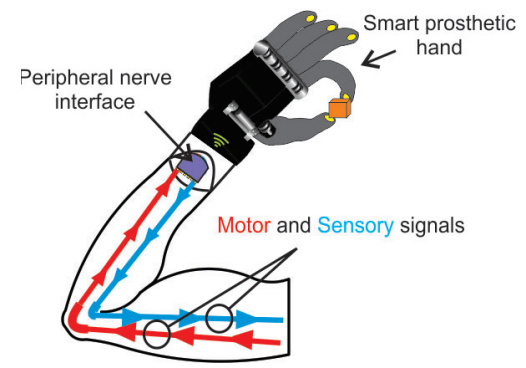

Figure 1: Peripheral nerve interfaces necessary for braincontrolled prostheses.

\section{DESIGN}

The LACE is fabricated from thin-film biocompatible polymer Parylene C (Figure 2), and incorporates 4 surface micromachined microfluidic channels $(250 \mu \mathrm{m} \mathrm{W} \times 20 \mu \mathrm{m} \mathrm{H})$ for targeting four fascicles in a rat sciatic nerve. Each microfluidic channel contains a pair of Pt electrodes $(300 \mu \mathrm{m} \mathrm{W} \times 1500 \mu \mathrm{m} \mathrm{L})$ for differential recording and/or stimulation. The cuff wraps around a nerve with the microfluidic outlets in direct contact with the circumference of the epineurium, and is held in position by etched serrations (Figure 2). The cuff is adjustable (1.1-1.5 mm diameter, $0.1 \mathrm{~mm}$ increments) to provide close fits to nerves of varying diameter. Ancillary components identified in Figure $2 \mathrm{~b}$ include a port for external fluidic connection, Parylene ribbon cable $(\sim 2 \mathrm{~cm}$ long) for external electrical connection, and a reference electrode external to the microfluidic channels.

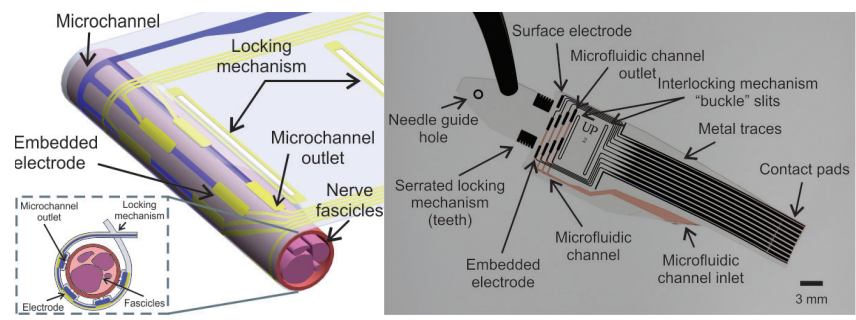

Figure 2: (a) Schematic of LACE for targeting individual fascicles within a nerve. Insert shows the cross-sectional view. (b) Fabricated $L A C E$ in which microfluidic channels are highlighted by the presence of photoresist.

\section{Fabrication}

Devices were fabricated on a silicon wafer for mechanical support during the microfabrication process (Figure 3). A $10 \mu \mathrm{m}$ Parylene base layer was first deposited by chemical vapor deposition. Sputtered Pt electrodes and leads (2000 ̊) were then patterned by a liftoff process with negative photoresist AZ 5214 EIR. A $10 \mu \mathrm{m}$ Parylene insulation layer was then deposited and selectively plasma-etched to expose electrodes and contact pads. Sacrificial photoresist AZ 4620 was spun to a height of $20 \mu \mathrm{m}$ and patterned to form the microfluidic channels. An $8 \mu \mathrm{m}$ Parylene layer 
was then deposited over the photoresist. Oxygen plasma etching created openings for microfluidic ports as well as the device cutout. Devices were released from the substrate and sacrificial photoresist was removed with an acetone soak. No stiction of Parylene structures was observed.
1. Deposit Parylene and $\mathrm{Pt}$ electrodes

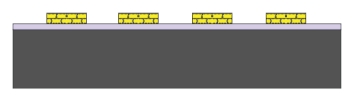

2. Deposit Parylene insulation and pattern sacrificial PR

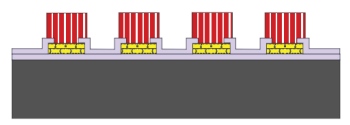

Silicon Parylene
3. Deposit Parylene

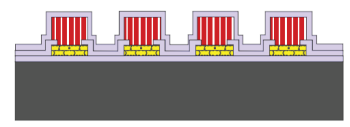

4. Etch and release devices from substrate

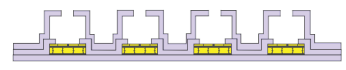

Figure 3: LACE fabrication process which utilizes standard surface micromachining techniques for Parylene.

Device level fluidic packaging consisted of a custom made polyurethane (PU) catheter (SAI Infusion Technologies) secured in place with MED 4210 silicone adhesive (Factor II Incorporated). A zero-insertion-force connector (ZIF, part no. 0514411072 Molex Inc) was used to establish reversible electrical connections to the cuff electrodes through the contact pads at the end of the integrated Parylene ribbon cable (Figure 4). The ZIF connector was mated to a flat flexible cable (FFC, part no. 050R10-102B, Parlex USA LLC) and a rigid $\mathrm{PCB}$ for external connection to the electrophysiological recording setup.

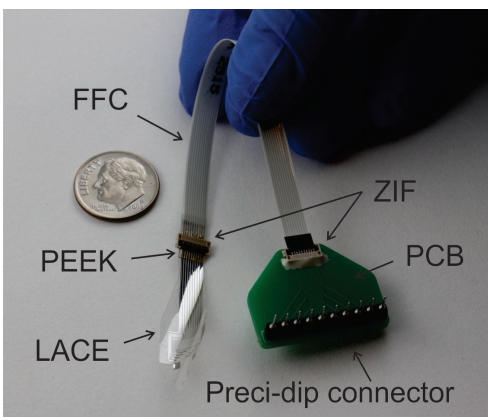

Figure 4: Cuff electrode with integrated Parylene cable is attached to a zero insertion force (ZIF) connector.

\section{METHODS}

Interlocking Mechanism

The cuff structure consisted of an elongated tapered tab that loops around the sciatic nerve, inserts into an etched slit and locks in place using etched serrated teeth (Figure 5a-c). A two-part locking mechanism was used to maintain the loop around the nerve, and includes an additional slit through which the tab is threaded to create a secure buckle structure (Figure $5 \mathrm{~d}$ ). The guide needle hole was utilized to pass a suture from the circular hole in the tab and facilitate guiding of the tab into the slits. Devices were wrapped and unwrapped around simulated agarose nerve phantoms and holding strength was evaluated under gentle handling conditions $(n>10)$. An agarose concentration of $1.37 \% \mathrm{wt} / \mathrm{wt}$ in water was selected since it has a compressive modulus equal to that of rabbit sciatic nerve $[12,13]$.
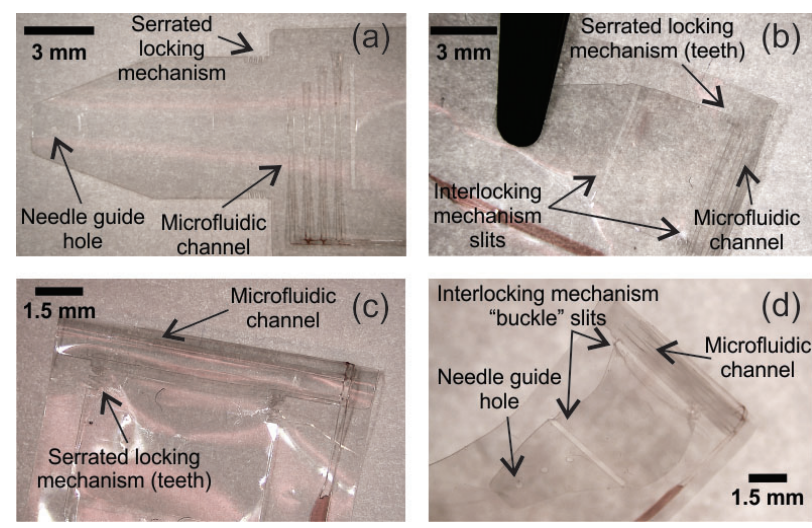

Figure 5: Prototype LACE interlocking mechanism test. (a) Flat $L A C E,(b-c)$ tab threaded through first slit and kept in place by serrated teeth, and (d) tab secured by threading through second slit to form the buckle locked structure.

\section{Microfluidic Channel Evaluation}

Evaluation of the microfluidic channels and fluidic connection was conducted by driving colored dye through the channels using an infusion pump (Harvard apparatus Infuse/Withdraw PHD 2000) and imaging the progression of the dye with a microscope (Caltex Scientific HD60T). The LACE device was submerged in distilled water under the microscope, and primed by flushing the channels with isopropyl alcohol and distilled water for 5 minutes each. With the LACE device in a flat or curled orientation, a solution of dilute green dye was infused into the LACE device at varied flow rates ranging from 5 to $2000 \mathrm{~nL} / \mathrm{min}$.

Maximum possible flow rate of the LACE device was determined by pumping dye through a LACE device wrapped around a nerve phantom, and observing which flow rates induced leaks or structural failure. Flow rates were increased from 300 $\mathrm{nL} / \mathrm{min}$ at increments of $100 \mathrm{~nL} /$ minute. Between each increment, the device was uncurled and infused with green dye in a flat orientation under the microscope in order to detect any small leaks that may have formed in the interior of the channels, then re-curled for the next flow rate.

Localization of fluid delivery was demonstrated by locking a LACE device around a simulated sciatic nerve phantom, then driving sodium fluorescein dye (Sigma F6377) through the microfluidic channels using the infusion method previously described at a flow rate of $50 \mathrm{~nL} / \mathrm{min}$.

\section{Electrode Evaluation}

Cyclic voltammetry (CV) and electrochemical impedance spectroscopy (EIS) were used to assess the stimulation and recording capabilities respectively of the cuff electrodes. Electrochemical characterization of the electrodes was performed with a Gamry Reference 600 potentiostat (Gamry Instruments, Warminster, PA). Prior to EIS measurement, CV was used to electrochemically clean the electrode surface and take initial measurements. LACE devices were immersed in $0.05 \mathrm{M} \mathrm{H}_{2} \mathrm{SO}_{4}$ with a constant stream of $\mathrm{N}_{2}$ in a three-electrode cell. The working electrode was cycled between -0.2 to $1.2 \mathrm{~V}$ with respect to an $\mathrm{Ag} / \mathrm{AgCl}(3 \mathrm{M} \mathrm{NaCl})$ reference, a potential range that is within the water window [14]. A $1 \mathrm{~cm}^{2} \mathrm{Pt}$ plate served as a counter electrode. Using a scan rate of $250 \mathrm{mV} / \mathrm{s}$, each electrode was cycled for 30 cycles. EIS was performed in phosphate buffer saline $(1 \times$ PBS $)$ at room temperature with an $\mathrm{AC}$ perturbation signal of $10 \mathrm{mV}(\mathrm{rms})$ in the frequency range of $1-10^{5} \mathrm{~Hz}$. As with $\mathrm{CV}$, an $\mathrm{Ag} / \mathrm{AgCl}(3 \mathrm{M}$ $\mathrm{NaCl}$ ) reference and $1 \mathrm{~cm}^{2} \mathrm{Pt}$ plate counter were used. 


\section{RESULTS}

\section{Interlocking Mechanism}

The locking mechanism was simple to implement and robust. A suture needle facilitated wrapping the device around an agarose nerve phantom of $1.2 \mathrm{~mm}$ diameter. The LACE was able to wrap tightly around the phantom without sliding of the device or damage to the simulated nerve. Once locked in place, the LACE did not disengage during fluidic and electrical testing, evidence of a secure lock. No damage to the cuff was observed despite repeatedly engaging and releasing the lock and curling and uncurling the LACE around the nerve phantom.

\section{Microfluidic Channel Evaluation}

No leakage or delamination was observed up to a flow rate of $2000 \mathrm{~nL} / \mathrm{min}$, with uniform flow through all fluidic channels and outlet ports in the flat configuration $(n=4)$. Figure 6 a displays a sequence of images showing simultaneous and equally distributed flow out of all four outlet ports in the flat orientation. Image analysis confirmed that flow rate through channels was uniform within $6 \%$ standard error. In the curled configuration, flow was achieved in 3 out of 4 channels which is sufficient for the in vivo application (Figure 6b). Flow through the distal channel was blocked by pinching of the horizontal feeder channel and can be mitigated by channel design changes such as: reduced feeder channel width and incorporation of Parylene support walls along the horizontal channel $[15,16]$. These changes have been incorporated and are being evaluated.

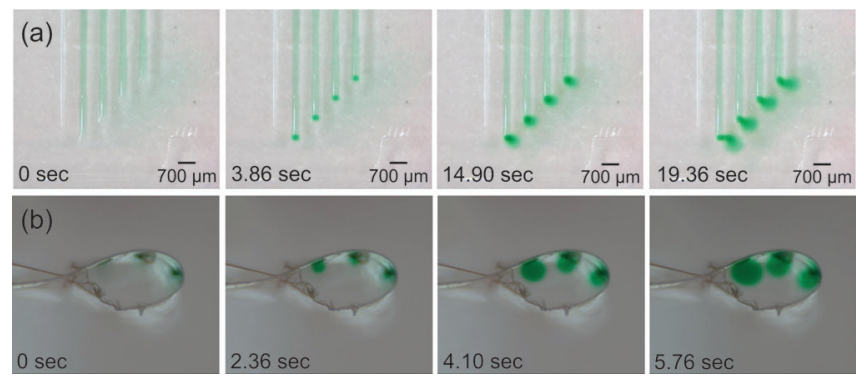

Figure 6: Sequential photographs of the infusion experiment at 830 $n L / m i n$ flow rate in a (a) flat device and (b) curled device. Image analysis using ImageJ software confirmed that flow rate through channels was uniform within $6 \%$ standard error.

During maximum flow rate testing, microfluidic channel inlet failure occurred at a rate of $1000 \mathrm{~nL} / \mathrm{min}$. The leak appeared to be from delamination between Parylene layers. A safe flow rate of 900 $\mathrm{nL} / \mathrm{min}$ can be achieved by the LACE device, which greatly exceeds the typical epineural space drug delivery rate of $17 \mathrm{~nL} / \mathrm{min}$ that will be employed in our studies [17]. Localized drug delivery was achieved with the LACE as shown in Figure 7. In on-going testing the agarose nerve will then be imaged using a fluorescent microscope (Nikon LV100; mercury $100 \mathrm{~W}$ lamp, Chiu Technical Corporation; B-2A filter cube) and digitally processed in ImageJ in order to correlate fluorescent signal with dye concentration.

\section{Electrode Evaluation}

The expected standard voltammogram for Pt immersed in sulfuric acid was achieved for all electrodes. Distinct peaks of current in the cyclic voltammogram result from the process of hydrogen atoms adsorption to and desorption from the electrode surface were observed as shown in Figure 8 . These peaks can be integrated with respect to the scan rate to calculate the electroactive surface area (ESA). In equation (1), $\mathrm{Q}_{\mathrm{H}}$ is the hydrogen desorption charged measured via $\mathrm{CV}$ and $210 \mu \mathrm{C} / \mathrm{cm}^{2}$ is the value for the characteristic charge density associated with the monolayer of hydrogen atoms adsorbed to polycrystalline Pt [18]. Stimulation electrodes can be characterized by their cathodal charge storage capacity (CSC), which is calculated from the time integral of the cathodic current in a cyclic voltammogram per unit geometric surface area (GSA) [19] as shown in equation (2).

$$
\begin{gathered}
E S A=\frac{Q_{H}}{210 \mu C_{c m^{2}}} \\
C S C=\frac{Q_{\text {cathodic }}}{G S A}
\end{gathered}
$$

The cyclic voltammogram obtained for all electrodes tested indicated a clean electrode surface. The ESA calculated was $635,714 \mu \mathrm{m}^{2}$, which is different from the GSA of $450,000 \mu \mathrm{m}^{2}$ due to surface topology. The CSC was $1.06 \pm 0.06 \mathrm{mC} / \mathrm{cm}^{2}$ and the roughness factor, defined as the ratio of ESA to GSA was $1.4(\mathrm{n}=$ 6). No bubble generation was observed during the CV, which is important for safe electrical stimulation.

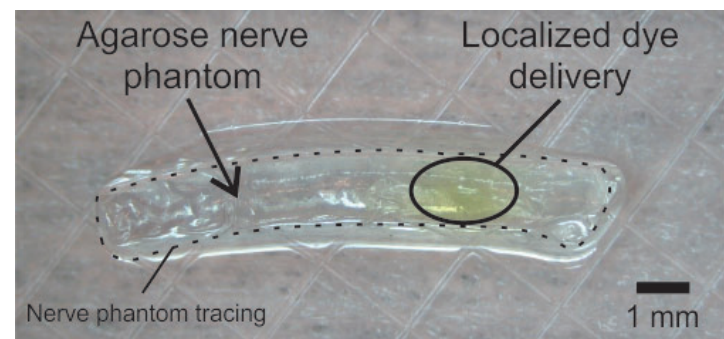

Figure 7: 1.37\% (w/w) agarose nerve phantom after localization testing with fluorescein dye.

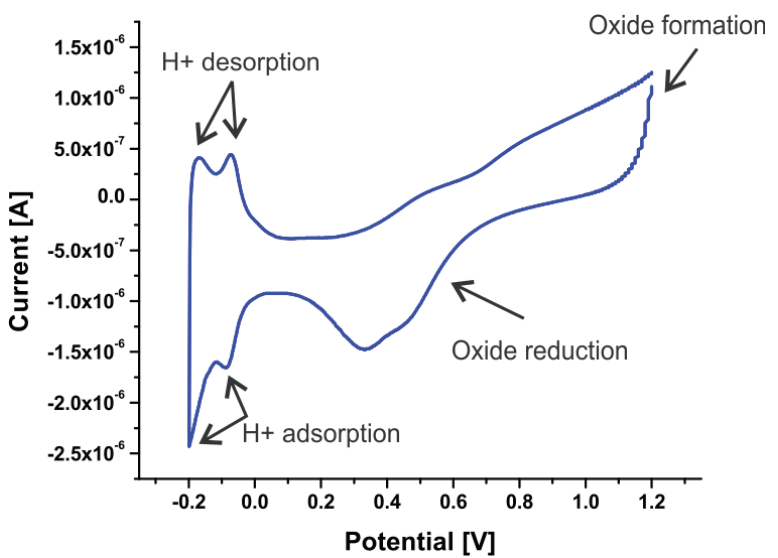

Figure 8: Cyclic voltammetry in $\mathrm{H}_{2} \mathrm{SO}_{4}$ of single recording site yielded standard Pt electrode characteristics.

EIS measurements on cuff electrodes yielded an electrical impedance of $1.8 \pm 0.11 \mathrm{k} \Omega$ and a phase of $-56.4 \pm 1.0^{\circ}$ at $1 \mathrm{kHz}$ demonstrating low variation between electrodes $(n=6)$. A representative EIS measurement for a single electrode site post CVclean is shown in Figure 9. Recorded impedances are expected to provide adequate neural recording potential. Baseline EC measurements on cuff electrodes demonstrated suitable electrode properties for peripheral nerve recordings and stimulation.

\section{CONCLUSION}

We developed a peripheral nerve interface that combines both electrodes and microfluidic channels in an adjustable cuff sized to interface with rat sciatic nerve. The LACE device uses a robust and simple locking mechanism that is adjustable for close contact with nerves of varying diameters. Parylene $\mathrm{C}$ was selected as the 
structural material for its biocompatibility and improved mechanical matching to tissue.

We demonstrated fully functional cuffs having low electrode impedances of $<2 \mathrm{k} \Omega(1 \mathrm{kHz})$ to help reduce susceptibility to noise pickup from sources such as muscle outside the cuff or recording equipment [20]. Controlled microfluidic infusion at $<\mu \mathrm{L} / \mathrm{min}$ flow rates and localized drug delivery was achieved, which is important to generate specific drug gradients necessary for guiding the axons into the microchannels. Future work entails characterization of modified fluidic channels to allow uniform flow through all channels in the curled orientation in order to target each fascicle inside the sciatic nerve, long-term characterization of electrodes, and preparation for in vivo animal testing.

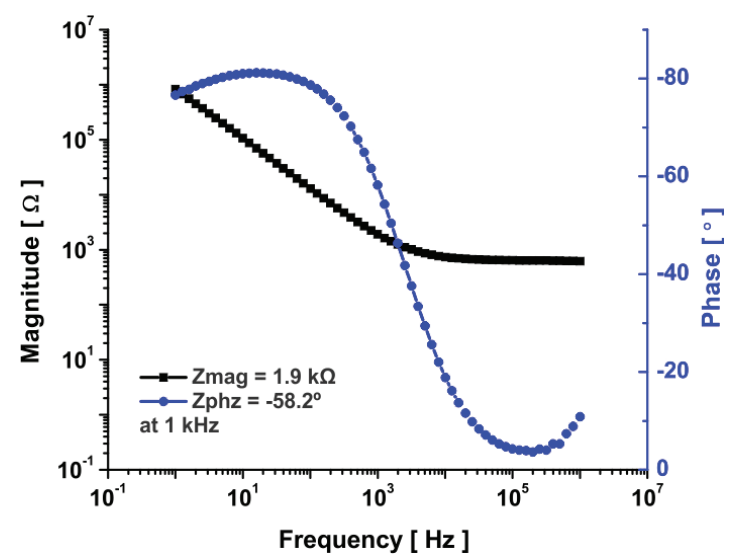

Figure 9: EIS in PBS of single recording site showed a low impedance $(<2 \mathrm{k} \Omega)$ at $1 \mathrm{kHz}$.

\section{ACKNOWLEDGEMENTS}

This work was supported under DARPA cooperative agreement HR0011-15-2-0006, Viterbi School of Engineering Ph. D. Merit Fellowship (AC), and USC Provost Fellowships (JY and TH). The authors would like to thank Dr. Donghai Zhu and the members of the USC Biomedical Microsystems Laboratory for their assistance with this project. Travel support was provided by OAI. An OAI hybralign series 200 mask aligner was used for processing of Parylene devices.

\section{REFERENCES}

[1] X. Navarro, T. B. Krueger, N. Lago, S. Micera, T. Stieglitz, and P. Dario, "A critical review of interfaces with the peripheral nervous system for the control of neuroprostheses and hybrid bionic systems," Journal of the Peripheral Nervous System, vol. 10, pp. 229-258, 2005.

[2] T. Stieglitz, M. Schuetter, and K. P. Koch, "Implantable biomedical microsystems for neural prostheses," Engineering in Medicine and Biology Magazine, IEEE, vol. 24, pp. 58-65, 2005.

[3] S. Micera, M. C. Carrozza, L. Beccai, F. Vecchi, and P. Dario, "Hybrid bionic systems for the replacement of hand function," Proceedings of the IEEE, vol. 94, pp. 1752-1762, 2006.

[4] P. M. Rossini, S. Micera, A. Benvenuto, J. Carpaneto, G. Cavallo, L. Citi, et al., "Double nerve intraneural interface implant on a human amputee for robotic hand control," Clinical neurophysiology, vol. 121, pp. 777-783, 2010.

[5] D. J. Tyler and D. M. Durand, "Functionally selective peripheral nerve stimulation with a flat interface nerve electrode," Neural Systems and Rehabilitation Engineering, IEEE Transactions on, vol. 10, pp. 294-303, 2002.
[6] T. Suzuki, N. Kotake, K. Mabuchi, and S. Takeuchi, "Flexible Regeneration-type Nerve Electrode with Integrated Microfluidic Channels," in Microtechnologies in Medicine and Biology, 2006 International Conference on, 2006, pp. 303-305.

[7] T. Stieglitz, H. Beutel, and J.-U. Meyer, "A flexible, lightweight multichannel sieve electrode with integrated cables for interfacing regenerating peripheral nerves," Sensors and Actuators A: Physical, vol. 60, pp. 240-243, 1997.

[8] B. Rydevix, M. D. Brown, T. Ehira, and C. Nordborg, "Effects of Collagenase on Nerve Tissue: An Experimental Study on Acute and Long-Term Effects in Rabbits," Spine, vol. 10, pp. 562-566, 1985.

[9] L. Isaacson, B. Saffran, and K. Crutcher, "Nerve growth factor-induced sprouting of mature, uninjured sympathetic axons," Journal of Comparative Neurology, vol. 326, pp. 327336, 1992.

[10] W.-C. Liao, Y.-J. Wang, M.-C. Huang, and G.-F. Tseng, "Methylcobalamin facilitates collateral sprouting of donor axons and innervation of recipient muscle in end-to-side neurorrhaphy in rats," PloS one, vol. 8, p. e76302, 2013.

[11] P. Haninec, R. Kaiser, and P. Dubový, "A Comparison of collateral sprouting of sensory and motor axons after end-toside neurorrhaphy with and without the perineurial window," Plastic and reconstructive surgery, vol. 130, pp. 609-614, 2012.

[12] M.-S. Ju, C.-C. K. Lin, J.-L. Fan, and R.-J. Chen, "Transverse elasticity and blood perfusion of sciatic nerves under in situ circular compression," Journal of biomechanics, vol. 39, pp. 97-102, 2006.

[13] V. Normand, D. L. Lootens, E. Amici, K. P. Plucknett, and P. Aymard, "New insight into agarose gel mechanical properties," Biomacromolecules, vol. 1, pp. 730-738, 2000.

[14] D. Zhan, J. Velmurugan, and M. V. Mirkin, "Adsorption/desorption of hydrogen on Pt nanoelectrodes: Evidence of surface diffusion and spillover," Journal of the American Chemical Society, vol. 131, pp. 14756-14760, 2009.

[15] C. H. Mastrangelo and G. Saloka, "A dry-release method based on polymer columns for microstructure fabrication," in Micro Electro Mechanical Systems, 1993, MEMS'93, Proceedings An Investigation of Micro Structures, Sensors, Actuators, Machines and Systems. IEEE., 1993, pp. 77-81.

[16] T.-J. Yao, X. Yang, and Y.-C. Tai, "BrF 3 dry release technology for large freestanding parylene microstructures and electrostatic actuators," Sensors and Actuators A: Physical, vol. 97, pp. 771-775, 2002.

[17] G. Cirillo, M. R. Bianco, A. M. Colangelo, C. Cavaliere, L. Zaccaro, L. Alberghina, et al., "Reactive astrocytosis-induced perturbation of synaptic homeostasis is restored by nerve growth factor," Neurobiology of disease, vol. 41, pp. 630-639, 2011.

[18] A. N. Frumkin, "Hydrogen overvoltage and adsorption phenomena -- 2," Advances in Electrochemistry and Electrochemical Engineering, vol. 3, pp. 287-391, 1962.

[19] S. F. Cogan, "Neural stimulation and recording electrodes," Annu. Rev. Biomed. Eng., vol. 10, pp. 275-309, 2008.

[20] G. Loeb and R. Peck, "Cuff electrodes for chronic stimulation and recording of peripheral nerve activity," Journal of neuroscience methods, vol. 64, pp. 95-103, 1996.

\section{CONTACT}

*E. Meng, tel: +1-213-821-3949; ellis.meng@usc.edu 